\title{
Preoperative Uterine Artery Embolization for Huge Prolapsed Pedunculated Submucosal Fibroid
}

\author{
Chinatsu Koiwai, Satoshi Ichigo, Hiroshi Takagi, Hiroyuki Kajikawa, Kazutoshi Matsunami, and Atsushi \\ Imai* \\ Department of Obstetrics and Gynecology, Matsunami General Hospital, Japan
}

Received: November 01, 2017; Published: November 08, 2017

*Corresponding author: Atsushi Imai, MD Institute of Endocrine Related Cancer, Matsunami General Hospital, Kasamatsu, Gifu 5016062, Japan

\section{Opinion}

Uterine fibroids (myomas) are very common affecting more than $60 \%$ of women over the age of 45 years [1,2]. Although many fibroids are asymptomatic, some may cause exhausting symptoms including heavy menstrual bleeding and pelvic pain. Submucosal fibroids, in particular pedunculated fibroids prolapsed into the vagina, are associated with heavy uterine bleeding and subsequent heavy anemia. The management of prolapsed pedunculated fibroid is determined by the origin and thickness of the pedicle, size, and its location. Uterine artery embolization (UAE) is increasingly being applied as a minimally invasive treatment alternative to surgery for the reduction of uterine fibroid symptoms [3]. UAEinduced depletion of blood flow through the whole myometrium and endometrium may be a useful adjunct to surgery for massive fibroids to reduce intraoperative blood loss [4].

\section{Case 1}

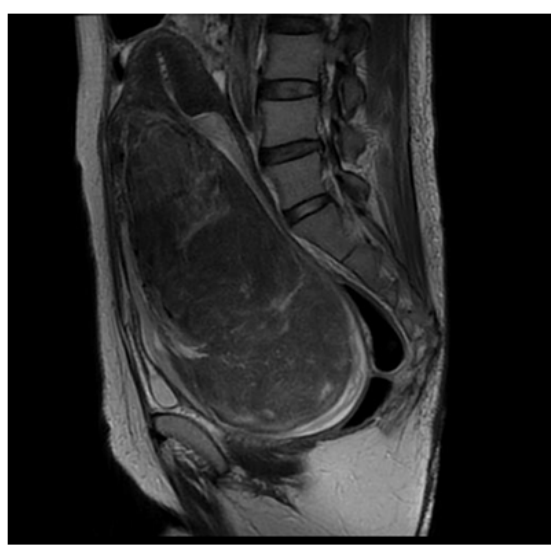

Figure 1a: Large pedunculated submucosal fibroid prolapsed into the vagina in 50-year-old women. Sagittal T2-weighted MR images show a solid mass occupying vaginal cavity (arrow head) and atrophic normalappearing uterus was visible (arrow). Pathologically, the tumor was diagnosed as a leiomyoma.
A 50 year old woman, G3P3, had a 1-year history of intermittent vaginal bleeding and progressive anemia. Physical examination revealed an ellipse of semi-solid; smooth surfaced mass occupying the vaginal cavity and hemoglobin level was remarkably decreasing (1.2 g/dl). Transfusion therapy was started immediately and intravenous iron prescribed to optimize her hemodynamic state. MRI detected a vaginal heterogenous mass $10 \times 15 \times 11 \mathrm{~cm}$, with regular edges and a vascular pedicle (Figure 1a). The mass seemed to dilate cervix up to the uterine fundus. Consideration of the inability to reach the pedicle through the vagina and the heavy vascularization of the fibroid led to the surgical approach through abdominal. The patient was put off UAE to reduce the blood supply, within 24 hours, with a surgical excision of $70 \%$ of the fibroid vaginally and subsequent simple abdominal hysterectomy and bilateral salpingo-oophorectomy.

Case 2

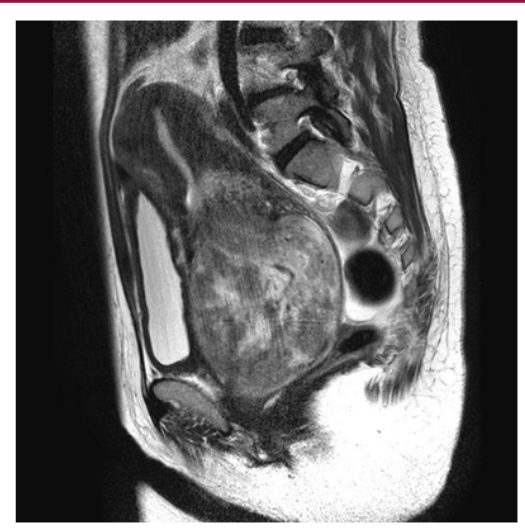

Figure 1b: Large pedunculated submucosal fibroid prolapsed into the vagina in 53-year-old women. Sagittal T2-weighted MR images show a solid mass occupying vaginal cavity (arrow head) and atrophic normalappearing uterus was visible (arrow). Pathologically, the tumor was diagnosed as a leiomyoma. 
A 53-year-old woman, G2P2 (2 cesarean sections), was referred to us complaining of significant vaginal discharge for 2 years. Pelvic examination detected an over man's fist sized, well-circumscribed mass in the vagina. Laboratory examinations revealed hemoglobin of $4.5 \mathrm{~g} / \mathrm{dl}$. MRI detected a margin-free vaginal mass $8 \times 9 \times 13 \mathrm{~cm}$, with a highly vascular pedicle (Figure $1 \mathrm{~b}$ ). The patient was submitted to transfusion therapy, UAE proceeding later with subsequent total abdominal hysterectomy with salpingo-oophorectomy within 48 hours.

For pedunculated fibroids prolapsed into the vagina, the most frequently chosen approach includes vaginal twisting or ligation and excision, morcellation of large fibroids has been used, with or without hysteroscopically assistance techniques [3]. Like our cases, due to the risk of excessive blood loss because of its size, the great feeding vascularization and the thick pedicle of the fibroid, the vaginal approach was limited, preoperative adjunct UAE before the surgical approach may be attractive to minimize blood loss. The subsequent vaginal morcellation and abdominal hysterectomy can be performed in a blood supply-depleted field. Although of smaller dimension, and therefore our case needed special management.
Although there is only two case of similar prolapsed fibroid in the literature [5,6], UAE may be very attractive before extracting large fibroids prolapsed into the vagina as a safe and minimally invasive technique.

\section{References}

1. Okolo S (2008) Incidence, aetiology and epidemiology of uterine fibroids. Best Pract Res Clin Obstet Gynaecol 22(4): 571-588.

2. Borah B, Yao X, Laughlin-Tommaso S, Heien H, Stewart E (2017) Comparative effectiveness of uterine leiomyoma procedures using a large insurance claims database. Obstet Gynecol 130(5): 1047-1056.

3. van der Kooij S, Ankum W, Hehenkamp W (2012) Review of nonsurgical/ minimally invasive treatments for uterine fibroids. Curr Opin Obstet Gynecol 27(6): 368-375.

4. Ngeh N, Belli A, Morgan R and Manyonda I (2004) Pre-myomectomy uterine artery embolisation minimises operative blood loss. Brit J Obstet Gynecol 111(10): 1139-1140.

5. Serradilla L, Gómez-Ríos M, CN (2011) Embolization before surgery of a large pedunculated submucosal myoma prolapsed into the vagina. Acta Obstet Gynecol Scand 90: 554-555.

6. Usta I, Hobeika E, Nassar A (2005) A tale of 2 pedunculated myomas. Am J Obstet Gynecol 193(5): 1753-1755.

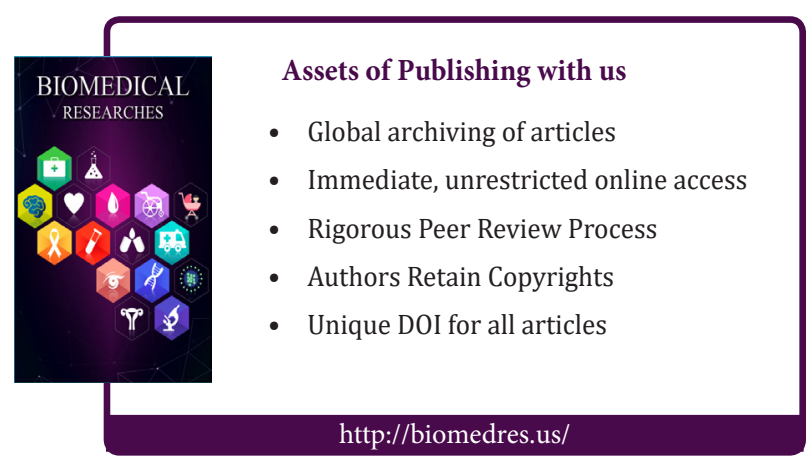

